\title{
LAS ORQUÍDEAS DEL PARQUE NACIONAL BARRA HONDA, GUANACASTE, COSTA RICA
}

\author{
Diego Bogarín ${ }^{1,2} \&$ Franco Pupulin ${ }^{1}$ \\ ${ }^{1}$ Jardín Botánico Lankester, Universidad de Costa Rica, P.O. Box 1031-7050 Cartago, Costa Rica, A.C \\ ${ }^{2}$ Autor para correspondencia: dbogarin@cariari.ucr.ac.cr
}

Palabras Clave: áreas protegidas, Costa Rica, estudios florísticos, Orchidaceae, Parque Nacional Barra Honda

\begin{abstract}
Introducción
Los trabajos florísticos constituyen fuentes de información de suma importancia pues entre otros aspectos, determinan el número de especies presentes en una región y permiten su correcta identificación (Atwood 1987, Pupulin 1998). La carencia de este tipo de trabajos dificulta futuras investigaciones en los diversos planos de la biología, la conservación y la educación ambiental. Costa Rica posee uno de los sistemas de áreas de conservación más importantes a nivel mundial. Cerca del $25 \%$ del territorio se encuentra protegido en unas 155 áreas silvestres, reservas biológicas y parques nacionales (Boza 1986). A pesar de esto, el aporte de los estudios florísticos al conocimiento de las especies de orquídeas que habitan en dichas áreas ha sido escazo y los inventarios respaldados por especímenes en herbarios son una excepción (Atwood 1987, Pupulin 1998, Gómez-Laurito \& R. Ortiz 2004). La mayoría de las áreas protegidas en Costa Rica carecen de esta información, la cual se encuentra muchas veces dispersa en registros de herbarios locales o extranjeros (Jiménez \& Grayum 2002, Pupulin 2003).
\end{abstract}

\section{Materiales y métodos}

El Parque Nacional Barra Honda (PNBH) se ubica en el cantón de Nicoya, provincia de Guanacaste (Boza 1986) (fig. 1). El interés de crear este parque surgió como una medida para proteger el sistema de cavernas de la región de Nicoya, originado por los eventos geológicos desde el Paleoceno Superior y Eoceno Inferior hasta el presente. Esta zona representa un área geológica muy importante para el país. (Mora 1978, Aguilar \& Denyer 2001, Jaccard et al. 2001). No obstante, la proteción de los recursos biológicos e hídricos es también otro de los objetivos se su creación. El PNBH comprende una extensión de 2.295 hectáreas donde predomina el bosque seco semideciduo del Pacífico Norte, caracterizado por una marcada estacionalidad climática (Tosi 1969, Janzen 1983). Históricamente, estos bosques han sufrido una fuerte intervención humana producto de la deforestación y el desarrollo de las actividades agropecuarias. Por otro lado, han sido bosques poco atractivos para los orquideólogos dada su baja diversidad de orquídeas en comparación con los bosques húmedos montanos y premontanos. Ante la fragilidad de estos ecosistemas de bosque seco, únicos en el país y la carencia de datos que permitan evaluar el número de especies y su identidad, es indispensable el aporte de los estudios florísticos para llevar a cabo acciones de conservación prioritarias en esta área (Boza 1986, Janzen 1983).

El presente estudio se realiza con base en recolectas de orquídeas en los diferentes sitios del PNBH. Durante el mes de Julio del 2005 y Febrero del 2006 se obtuvieron los datos que aquí se presentan. El material recolectado fue llevado a cultivo en las colecciones vivas del Jardín Botánico Lankester, Universidad de Costa Rica. Los testigos fueron depositados en las colecciones en alcohol del Jardín Botánico Lankester (JBL) y en el Herbario Nacional de Costa Rica (CR).

\section{Resultados}

En la primera etapa del estudio se determinó que la flora de orquídeas está compuesta por 26 especies en 23 géneros. Los géneros más representados son Epidendrun (3 especies), Scaphyglottis y Specklinia (ambos con 2 especies). Los demás 


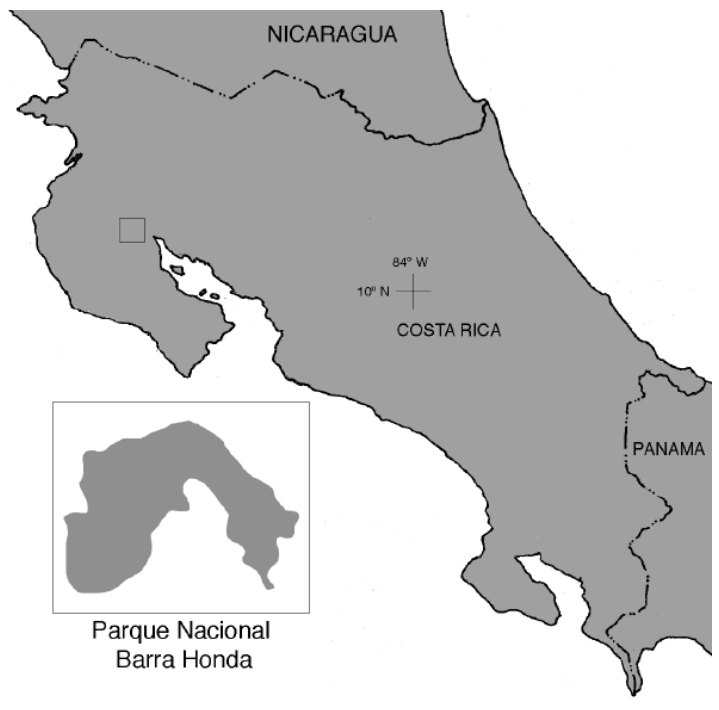

FIgURA 1. Ubicación del Parque Nacional Barra Honda.

géneros $(88.5 \%)$ están respresentados en el parque por una sola especie. E1 $73 \%$ de las especies son epífitas y un $27 \%$ presenta un hábito terrestre (cuadro 1). El período de floración se concentra entre los meses de Noviembre a Abril, básicamente durante la estación seca.

\section{Discusión}

Algunas de las especies del PNBH tienen una amplia distribución a nivel nacional y habitan en toda la costa Pacífica, tanto en las zonas húmedas del sur como en las áreas secas estacionales del norte y parte del Valle Central. Estas especies son: Epidendrum coronatum Ruiz \& Pav., Epidendrum stamfordianum Bateman, Epidendrum vulgoamparoanum Hágsater y Scaphyglottis stellata Lodd. ex Lindl. Tres especies, Pleurothallis quadrifida (Lex.) Lindl., Scaphyglottis micrantha (Lindl.) Ames \& Correll y Sobralia decora Bateman presentan este tipo de distribución pero se les puede encontrar también en la parte húmeda del Caribe norte. Además, Brassavola nodosa (L.) Lindl., Trigonidium egertonianum Bateman ex Lindl., Catasetum maculatum Kunth, Specklinia grobyi (Bateman ex Lindl.) F. Barros y Dimerandra emarginata (G.Mey.) Hoehne, pueden distribuirse, tanto a lo largo de toda la vertiente Pacífica como en la vertiente Caribe. En el PNBH estas especies se pueden observar con relativa frecuencia en los bosques secundarios poco alterados.

Dentro de la región biogeográfica del Pacífico Norte, existe otro grupo de especies que se encuentran en las zonas estacionales húmedas y secas hacia el norte de la cuenca del río Grande de Tárcoles y alcanzan sitios estacionales cercanos al Valle Central. Este grupo presenta su límite de distribución sureño en las áreas circunvecinas al Cerro Turrubares, la cuenca del Tárcoles y el río Candelaria y su hábitat corresponde más con los bosques estacionales secos del norte (Jiménez \& Grayum 2002). Dentro de este grupo encontramos Barkeria obovata (C. Pres1) Christenson, Cohniella cebolleta (Jacq.) Christenson, Cyrtopodium paniculatum (Ruiz \& Pav.) Garay, Encyclia cordigera (Kunth) Dressler, Laelia rubescens Lindl. y Trichosalpinx blaisdellii (S. Watson) Luer. Estos datos son apoyados por estudios florísticos en la región central y sur de la costa Pacífica, donde no se reporta la presencia de estas especies (Pupulin 1998, Weber et. al 2001, Jiménez $\&$ Grayum 2002). Sin embargo, esta misma área biogeográfica es el límite para algunas especies del Pacífico Central y Sur que crecen en las áreas más humedas y poco estacionales y no alcanzan una distribución más norteña. Este patrón es observado en la flora de orquídeas del PNBH y especies como Specklinia corniculata (Sw.) Steud., Aspasia epidendroides Lindl., Ionopsis satyrioides (Sw.) Rchb.f., Trizeuxis falcata Lindl., Prosthechea abbreviata (Schltr.) W.E.Higgins, Campylocentrum multiflorum Schltr. y C. micranthum Lindl., entre otras, se distribuyen al sur de la costa Pacífica sin alcanzar las áreas estacionales del Pacífico Norte (Pupulin 1998, Jiménez \& Grayum 2002).

En el PNBH se protege la flor nacional, Guarianthe skinneri (Bateman) Dressler \& W.E. Higgins. Su distribución es también amplia a lo largo del territorio nacional, sin embargo sus poblaciones silvestres han sido reducidas por la deforestación y excesiva recolecta. Algunas poblaciones se encuentran en el PNBH sin embargo es difícil obsevarlas aparentemente por la extracción ilegal dentro del parque.

Siete especies con hábito terrestre se encuentran en el parque. Durante el período seco, todas las plantas de estas especies presentan un comportamiento deciduo, perdiendo sus hojas durante los 
CuAdro 1. Hábito y distribución general de las orquídeas del Parque Nacional Barra Honda.

\begin{tabular}{|c|c|c|c|c|}
\hline Género y especie & Hábito & Amplia & Influencia & Pacífico \\
\hline 1. Barkeria obovata (C. Presl) Christenson & $\mathrm{E}$ & & & $x$ \\
\hline 2. Beloglottis costaricensis (Rchb.f.) Schltr. & $\mathrm{T}$ & & $x$ & \\
\hline 3. $\quad$ Brassavola nodosa (L.) Lindl. & $\mathrm{E}$ & $\mathrm{x}$ & & \\
\hline 4. $\quad$ Catasetum maculatum Kunth & $\mathrm{E}$ & $x$ & & \\
\hline 5. Cohniella cebolleta (Jacq.) Christenson & $\mathrm{E}$ & & & $x$ \\
\hline 6. Cyrtopodium paniculatum (Ruiz \& Pav.) Garay & $\mathrm{T}$ & & & $x$ \\
\hline 7. Dimerandra emarginata (G.Mey.) Hoehne & $\mathrm{E}$ & $\mathrm{x}$ & & \\
\hline 8. Encyclia cordigera (Kunth) Dressler & $\mathrm{E}$ & & & $x$ \\
\hline 9. $\quad$ Epidendrum coronatum Ruiz \& Pav. & $\mathrm{E}$ & & $\mathrm{x}$ & \\
\hline 10. Epidendrum stamfordianum Bateman & $\mathrm{E}$ & & $\mathrm{x}$ & \\
\hline 11. Epidendrum vulgoamparoanum Hágsater & $\mathrm{E}$ & & $\mathrm{x}$ & \\
\hline 12. Guarianthe skinneri (Bateman) Dressler \& W.E. Higgins & E & & $x$ & \\
\hline 13. Habenaria macroceratitis Willd. & $\mathrm{T}$ & $x$ & & \\
\hline 14. Laelia rubescens Lindl. & $\mathrm{E}$ & & & $x$ \\
\hline 15. Malaxis aurea Ames & $\mathrm{T}$ & & $\mathrm{x}$ & \\
\hline 16. Oeceoclades maculata (Lindl.) Lindl. & $\mathrm{T}$ & $\mathrm{x}$ & & \\
\hline 17. Palmorchis sp. & $\mathrm{T}$ & & & \\
\hline 18. Pleurothallis quadrifida (Lex.) Lindl. & E & $x$ & & \\
\hline 19. Scaphyglottis micrantha (Lindl.) Ames \& Correll & E & $\mathrm{x}$ & & \\
\hline 20. Scaphyglottis stellata Lodd. ex Lindl. & E & & $\mathrm{x}$ & \\
\hline 21. Sobralia decora Bateman & E & $\mathrm{x}$ & & \\
\hline 22. Specklinia grobyi (Bateman ex Lindl.) F. Barros & $\mathrm{E}$ & $\mathrm{x}$ & & \\
\hline 23. Specklinia microphylla (A. Rich \& Galeotti) Pridgeon \& M.W.Chase & E & & $x$ & \\
\hline 24. Sarcoglottis sp. & $\mathrm{T}$ & & & \\
\hline 25. Trichosalpinx blaisdellii (S. Watson) Luer & E & & & $x$ \\
\hline 26. Trigonidium egertonianum Bateman ex Lindl. & E & $x$ & & \\
\hline
\end{tabular}

$\mathrm{E}=$ Epífita; $\mathrm{T}=$ Terrestre

meses secos. Únicamente Palmorchis sp. y Oeceoclades maculata (Lindl.) Lindl. conservan sus hojas durante todo el año. Las plantas de Cyrtopodium paniculatum crecen en áreas rocosas con materia orgánica en bosques secundarios. Habenaria macroceratitis Willd. se encuentra a orillas de caminos en zonas expuestas y alteradas o en el bosque secundario. Sarcoglottis sp., una especie aún no identificada, crece en grandes colonias en el interior de los bosques secundarios y zonas rocosas en sitios con sombra. Las poblaciones presentan cierta variación en la coloración de las hojas y las flores. Beloglottis costaricensis (Rchb.f.) Schltr., una especie poco conocida y casi nunca recolectada es fácil de observar en el PNBH usualmente en poblaciones mezcladas con Sarcoglottis sp.

Dentro de los hallazgos más importantes está la presencia de Malaxis aurea Ames, una especie poco común, conocida únicamente del bosque húmedo premontano. En el PNBH se encuentra en zonas de bosque secundario en suelos rocosos con materia orgánica. Este hallazgo representa un dato ecológico nuevo para esta especie. Una especie de Palmorchis todavía no identificada es también un nuevo registro en términos ecológicos para el sitio y podría tratarse de una especie no descrita (Dressler, com. pers. 2005). La especie exotica y recién naturalizada, Oeceoclades maculata es común en ciertas áreas del PNBH y forma 
colonias a lo largo de áreas de bosque secundario. $\mathrm{Su}$ presencia se reporta para las áreas secas del Parque Nacional Santa Rosa y ha sido recolectada en el Pacífico Central y Sur (Dressler 2003).

Las acciones futuras del proyecto se concentran en evaluar otras áreas del PNBH pues es posible encontrar algunas especies que habitan en la misma región biogeográfica del bosque seco. Además, el material disponible de Specklinia microphylla (A. Rich \& Galeotti) Pridgeon \& M.W.Chase y Trichosalpinx blaisdelli (S. Watson) Luer, requiere de un estudio taxonómico detallado, siendo posible que estos nombres no estén correctamente aplicados al material encontrado. El presente estudio proporciona nueva información sobre los ecosistemas de bosque seco e intenta explicar la flora de orquídeas del PNBH basandose en la distribución general de las especies a nivel nacional. Esta información puede ser aprovechada por las demás áreas protegidas dentro el mismo ecosistema, las cuales comparten algunas especies que alcanzan una distribución más amplia. De esta manera se pretende iniciar un sistema que permita fortalecer el conocimiento y la protección de la flora de orquídeas de la región del bosque seco de Costa Rica.

Agradecimientos. Un agradecimiento especial a Jorge Granados, Director del Parque Nacional Barra Honda, a Elmer García, Federico Paniagua, el personal del parque y a los guías Reynaldo Orias, Franklin Villalobos y Mariano Quesada por su colaboración en el campo. A Darwin Initiative, DEFRA, Reino Unido, en el proyecto "Conservation and Monitoring of Meso-American Orchids" (ref. 14-001), por su aporte al estudio de las orquídeas en áreas protegidas de Costa Rica. Las recolectas de material fueron realizadas bajo el Pasaporte Científico $\mathrm{N}^{\circ} 010925$, resolución $\mathrm{N}^{\circ}$ 042-2005-OFAU, emitido por el Ministerio de Ambiente y Energía (MINAE).

\section{Literatura CitADA}

Aguilar, T \& P. Denyer. 2001. Una especie nueva de Euphyllia (Scleractinia: Caryophylliidae) en las calizas de Barra Honda (Paleógeno), Costa Rica. Rev. Biol. Trop. 49. Supl. 2: 195-201.

Atwood, J. T. 1987. The vascular flora of La Selva Biological Station, Costa Rica: Orchidaceae. Selbyana 10: 76-145.

Boza, M. A. 1986. Parques Nacionales Costa Rica National Parks. Fundación de Parques Nacionales, Costa Rica. Incafo, Madrid.

Dressler, R. 2003. Orchidaceae. Pp. 1-595 in: B.E. Hammel, M.H. Grayum, C. Herrera \& N. Zamora (eds.). Manual de Plantas de Costa Rica. Vol. III. OrchidaceaeZingiberaceae. Monogr. Syst. Bot. Missouri Bot. Gard. 93.

Gómez-Laurito, J. \& R. Ortiz. 2004. Lista con anotaciones de las angiospermas de la Reserva Biológica Alberto Brenes (microcuencas de los ríos San Lorenzo y San Lorencito), Costa Rica. Lankesteriana 4: 113-142.

Jaccard, S., M. Münster, P.O. Baumgartner, C.BaumgartnerMora \& P. Denyer. 2001. Barra Honda (Upper Paleocene- Lower Eocene) and El Viejo (CampanianMaastrichtian) carbonate platforms in the Tempisque area. Rev. Geol. Amér. Central 24: 9-28.

Jiménez, Q. \& M.H. Grayum. 2002. Vegetación del Parque Nacional Carara, Costa Rica. Brenesia. 57-58.

Janzen, D.H. (ed.) 1983. Costa Rican natural history. Chicago University, Chicago.

Mora, S. 1978. Estudio geológico de los Cerros Barra Honda y alrededores, cantón de Nicoya, provincia de Guanacaste, Costa Rica. Tesis de Bachillerato. San José, Universidad de Costa Rica. 173 p.

Pupulin, F. 1998. Orchid florula of Parque Nacional Manuel Antonio, Quepos, Costa Rica. Rev. Biol. Trop. 46 (4): 961-10317.

Pupulin, F. 2003. Orquídeas de Tapantí. Jardín Botánico Lankester, Universidad de Costa Rica.

Tosi, J. 1969. Mapa ecológico de Costa Rica. Centro Científico Tropical, San José, Costa Rica.

Weber, A., W. Huber, A. Weissenhofer, N. Zamora \& G. Zimmermann (eds.) 2001. An introductory field guide to the flowering plants of the Golfo Dulce rainforests, Costa Rica. Corcovado National Park and Piedras Blancas National Park ("Regenwald der Österreicher"). Stapfia 78: 1-464.

Diego Bogarín obtuvo su grado en Biología en la Universidad de Costa Rica. Actualmente es investigador del Jardín Botánico Lankester, interesado en la sistemática y taxonomía de Orchidaceae. Recientemente, desarrolla proyectos de conservación de orquídeas en areas protegidas de Costa Rica en conjunto con las autoridades gubernamentales. Comenzó en el 2005 como Darwin Initiative Project Implementation Officer para el proyecto "Conservación y monitoreo de orquídeas Meso-Americanas", en colaboración con el Royal Botanic Gardens, Kew.

Franco Pupulin es profesor de la Universidad de Costa Rica donde labora como investigador del Jardín Botánico Lankester. Tiene interés particular en la sistemática y evolución de la especies de las subtribus Oncidiinae y Zygopetalinae. Actualmente trabaja en varios proyectos monográficos sobre la flora de América Central. Es investigador asociado de Marie Selby Botanical Gardens y del Oakes Ames Orchid Herbarium de la Universidad de Harvard. 